\title{
Precise characterisation of exoplanet-host stars parameters
}

\author{
Sylvie Vauclair ${ }^{1}$ \\ ${ }^{1}$ Institut de Recherches en Astrophysique et Planetologie, \\ 14 avenue Edouard Belin, 31400, Toulouse, France \\ email: sylvie.vauclair@ast.obs-mip.fr
}

\begin{abstract}
Studying the internal structure of exoplanet-host stars compared to that of similar stars without detected planets is particularly important for the understanding of planetary formation. In this framework, asteroseismic studies represent an excellent tool for a better characterization of stars and for a precise determination of the stellar parameters like mass, radius, gravity, effective temperature. The detection of stellar oscillations is obtained with the same instruments as used for the discovery of exoplanets, both from the ground and from space, although the time scales are very different. Here I discuss some details about the characterization of exoplanethost stars from seismology and the importance of the helium and heavy element abundances in this respect.
\end{abstract}

Keywords. stars: abundances, stars: oscillations, stars: interiors

\section{Introduction}

Detailed studies of exoplanets need precise knowledge of the central stars of planetary systems. In this framework, the best way to obtain precise values of the stellar parameters is asteroseismology combined with spectroscopic observations.

The observations for stellar oscillations and exoplanet searches are done with the same instruments. In some cases, the same observations, analysed on different time scales, can lead to both planet detection and seismic studies. This was the case for the star $\mu$ Arae, observed with HARPS during eight nights in June 2004: these observations, aimed for asteroseismology, lead to the discovery of the exoplanet $\mu$ Arae d (Santos et al. 2004). Another case is that of the planet discovered around the extreme horizontal branch star V391 Peg (Silvotti et al. 2007), which was detected owing to the seismic period variations (the so-called "time delay method").

Generally speaking, the goals of the asteroseismology of exoplanets-host stars are to derive precisely their masses, radii, ages, evolutionary stages, and internal structure. At the present time, with a detailed seismic analysis, it is possible to obtain the masses and radii of solar-type stars with a precision of a few 1 percent, from both ground and space observations (C.f. Vauclair et al. 2008; Metcalfe et al. 2010).These precise values are important for the determination of the parameters of the planets. They can also be compared to those of the Sun and stars without detected planets, to obtain hints about the theories of planetary formation and migration.

\section{Basics of asteroseismology for slowly rotating solar-type stars}

In solar-type stars, acoustic waves are permanently created by the motions which occur in their outer layers, induced by convection and related processes. The waves are damped, but as other waves always appear, the stellar sphere globally behaves as a resonant cavity, and the oscillations can be treated, with a very good approximation, as standing waves. 
Each mode can be characterized with three numbers: the number of nodes in the radial direction, $n$, and the two tangential numbers $\ell$ and $m$, which appear in the development of the waves on the spherical harmonics:

$$
Y_{l}^{m}(\theta, \phi)=(-1)^{m} C_{l, m} P_{l}^{m}(\cos \theta) \exp (i m \theta)
$$

Several combinations of the oscillation frequencies are used to obtain more precise constraints on the stellar internal structure, like the "large separations", which are defined as the differences between two consecutive modes of the same $\ell$ number, and the "small separations", defined as:

$$
\delta \nu=\delta \nu_{n, l}-\nu_{n-1, l+2}
$$

For acoustic modes in a given star, the large separations are nearly constant, and their average value is approximately half the inverse of the stellar acoustic time, i.e. the time needed for the $l=0$ waves to travel along the whole radius. However detailed studies of these frequency separations as a function of the frequency itself, give evidence of characteristic modulations. They are related to the regions of partial reflection of the waves inside the stars, which occur for rapid variations of the sound velocity. The modulations of the small separations are directly related to the structure of the stellar cores (Roxburgh \& Vorontsov 1994; Roxburgh 2007; Soriano et al. 2007; Soriano \& Vauclair 2008), whereas those of the large separation give information on the stellar upper layers, like the regions of helium ionization, the bottom of the convective zones or the diffusion-induced helium gradient (Vauclair \& Théado 2004; Castro \& Vauclair 2006).

\section{Asteroseismology of exoplanet-host stars}

Aseroseismology of exoplanet-host stars is a useful tool to determine their internal structure and their behaviour with respect to stars without detected planets. Let us recall that, due to the present observation bias, many stars may very well host undetected planets. In this framework, the special characteristics of exoplanet-host stars compared to the other ones are more related to the migration process of the planets than to their mere existence.

The most important goals at the present time are the determination of masses, radii and ages of exoplanet-host stars with the best possible precision. When comparing the observed frequencies and frequency differences with the computed ones, one must take into account the details of the chemical composition.

Most exoplanet-host stars are overmetallic, compared to the other stars and to the Sun (e.g. Santos et al. 2005). This overmetallicity is not due to accretion of planetary material, as the accreted heavy matter falls down due to thermohaline convection induced by the unstable $\mu$-gradient (Vauclair 2004). It was present in the instersellar cloud out of which the stellar system formed, and this must be taken into account in the models.

A crucial unknown parameter, very important for the determination of the stellar characteristics, is the helium abundance. Unfortunately, helium is not directly observable in the spectra of solar-type stars. Contrary to what is often admitted, it cannot be safely related to the overmetallicity, as the helium versus metal enrichment may strongly vary inside a galaxy. For the Hyades, for example, the helium content is much smaller than could be expected from the metal enrichment (Lebreton et al. 2001).

When comparing the observed frequencies and frequency differences for a given star with those of different models computed with different helium contents, one find the same gravities (log g) with a precision better than 1 percent, very close radii and masses. On 


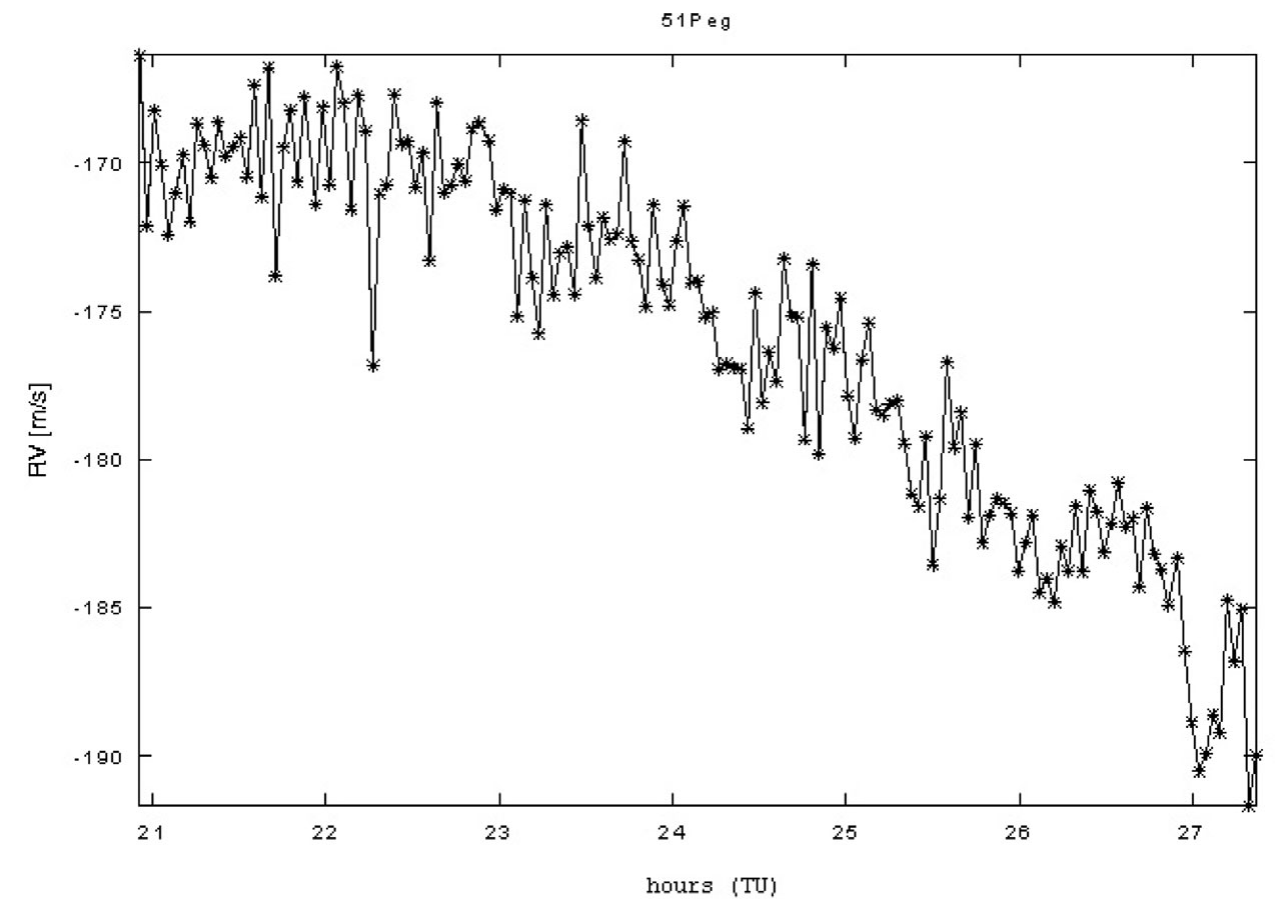

Figure 1. The star $51 \mathrm{Peg}$ has been observed for asteroseismology with the SOPHIE spectrometer, at Haute Provence Observatory, in August 2007 and 2010. The graph presents part of the radial velocity curve, obtained during one night. The solar-like oscillations are clearly visible. The overall decrease of the radial velocity during the night is the signature of the exoplanet discovered by Mayor and Queloz 1995

the other hand, ages may differ by large values. Detailed seismic analysis can help solving this question, with the help of spectroscopy, as has been proved for $\iota$ Hor (Vauclair et al. 2008) and $\mu$ Arae (Soriano \& Vauclair 2010).

Up to now four exoplanet-host stars have been observed and deeply analyzed from the ground on relatively long periods ( 8 or 9 consecutive nights) with the HARPS and SOPHIE spectrometers. They are: $\mu$ Arae (HARPS, 2004) (Bazot et al. 2005; Soriano \& Vauclair 2010); ८ Hor (HARPS, 2006) (Vauclair et al. 2008), 94 Cet (HARPS, 2007) and 51 Peg (SOPHIE, 2007 and 2010) (Bazot et al. 2011 in preparation, Figure 1). Several of them have been observed from space, but the most detailed analysis is that of the main COROT target HD52265 (Ballot et al. 2011).

The asteroseismology of exoplanets-host stars is a new field which will become more and more important in the near future.

\section{Conclusion}

From the few examples already available, asteroseismology has proved to be a powerful tool for determining stellar parameters, in particular for exoplanet-host stars. The scientific community is well prepared for future observations with on-going or planned projects. Space missions like KEPLER, and later on PLATO, are expected to give a large amount of new data for seismology, besides planet searches. Recent studies of solar-type stars with Kepler lead to radii determinations with a precision of 1 percent (Metcalfe et al. 2010). Meanwhile ground based instruments devoted to exoplanets like HARPS or SOPHIE can be used for seismology. All these observations will give the possibility of 
using the seismic tests with a precision never obtained before. They will lead to better parameters for exoplanet-host stars and help for a better understanding of planetary formation and evolution.

\section{References}

Bazot, M. \& Vauclair, S. 2004, A\&SA, 427, 965

Bazot, M., Vauclair, S., Bouchy, F., \& Santos, N. 2005, A\&A, 440, 615

Ballot, J., Gizon, L., et al. 2011 in preparation

Bouchy, F., Bazot, M., Santos, N., Vauclair, S., \& Sosnowska, D. 2005, AछAA, 440, 609

Castro, M. \& Vauclair, S. 2006, A\&A, 456, 611

Lebreton, Y., Fernandes, J., \& Lejeune, T. 2001, A\&A, 374, 540

Mayor, M. \& Queloz, D. 1995, Nature, 78, 355

Metcalfe, T. S., Monteiro, M. J. P.. F. G.., Thompson, M. J. et al. 2010, ApJ, 723, 1583

Roxburgh, I. W., \& Vorontsov, S. V. 1994, MNRAS, 267, 297

Roxburgh, I. W. 2007, MNRAS, 379, 801

Santos, N. C., Israelian, G., \& Mayor, M. 2004, A\&A, 415, 1153

Santos, N. C., Israelian, G., Mayor, M., Bento, J. P., Almeida, P. C., Sousa, S. G., \& Ecuvillon, A. $2005, A \& A, 437,1127$

Silvotti, R., Schuh, S., Janulis, R., et al. 2007, Nature, 449, 189

Soriano, M., Vauclair, S., Vauclair, G., \& Laymand, M. 2007, A\&A, 471, 885

Soriano, M. \& Vauclair, S. 2008, A\& $A, 488,975$

Soriano, M. \& Vauclair, S. 2010, $A \& A, 513,49$

Vauclair, S. 2004, ApJ, 605, 874

Vauclair, S. \& Théado, S. 2004, A\&A, 425, 179

Vauclair, S., Laymand, M., Bouchy, F., Vauclair, G., Hui Bon Hoa, A., Charpinet, S., \& Bazot, M. 2008, $A \& A$ A 482 , L5 